\title{
The effect of psychomotor therapy on mental health in in-patient schizophrenia treatment: A randomized, double-blind intervention study
}

\author{
Běla Hátlová ${ }^{1}$, Milena Adámková Ségárd ${ }^{1}$, Michel Probst ${ }^{2}$, Vlastimil Chytrý1 ${ }^{1}$, and Eva Chalupová1,* \\ ${ }^{I}$ Faculty of Education, Jan Evangelista Purkyně University in Ústí nad Labem, Ústí nad Labem, Czech Republic; and \\ ${ }^{2}$ Faculty of Movement and Rehabilitation Sciences, Catholic University of Leuven, Leuven, Belgium
}

Copyright: (C) 2020 B. Hátlová et al. This is an open access article licensed under the Creative Commons Attribution License (https://creativecommons.org/licenses/by/4.0/).

\begin{abstract}
Background: It has been shown that exercise programmes can influence mental health in patients with mental health disorders. Objective: The aim of this study was to verify the suitability of psychomotor therapy as a supporting method in the treatment of hospitalized patients with schizophrenia. Methods: In this randomized controlled doubleblind study 142 in-patients with schizophrenia (age: mean 50.2 years, $S D=6.5$, range 21-62) participated. In comparison with a control group $(n=60)$, the experimental group $(n=82)$ received an additional psychomotor therapy programme ( 3 times a week for a period of 14 weeks). The Brief Psychiatric Rating Scale (BPRS) was used to evaluate mental health status. Results: Participants in the psychomotor therapy demonstrated significant improvements for all subscales of the BPRS. Large effect sizes were found for subscales Anxiety/Depression, Energy, Thought Disturbance and Activation, and medium for Hostility. No significant difference between genders was found. Conclusion: The implementation of a psychomotor therapy programme in the treatment of hospitalized patients with schizophrenia has a significant positive effect on their mental health.
\end{abstract}

Keywords: BPRS, exercise, psychomotor therapy, schizophrenia

\section{Introduction}

Physical activity has a significant impact on mental well-being. Currently, physical activity is well accepted in our society, and this fact creates suitable situations for many clinical populations, such as patients with schizophrenia (Harvey, Hotopf, Overland, \& Mykletun, 2010; Mutrie \& Faulkner 2003; Richardson et al., 2005; Rosenbaum et al., 2016). Hölter (2011) mentioned that psychomotor therapy, a specific adapted physical activity for persons with mental health problems, can have a positive effect on their mental health. Patients are removed from their immersion in their own world by focusing on physical activities.

Schizophrenic disorders are generally characterized by a significant breakdown in thinking, misperception and blunted or flat affect and a lack of emotional reactivity. The senses of individuality, unity and autonomy, which are the essential functions of the personality, are

\footnotetext{
* Address for correspondence: Eva Chalupová, Department of Psychology, Faculty of Education, Jan Evangelista Purkyně University in Ústí nad Labem, Hoření 13, 40096 Ústí nad Labem, Czech Republic. E-mail: eva.chalupova@ujep.cz
}

affected. Most patients show impairment in the areas of concentration, attention, psychomotor learning, intentionality, and motivation (Gaebel \& Falkai, 1998). Loss of the boundaries of "oneself" is a typical sign of these patients. A clear consciousness and intellectual skills tend to be retained, even though specific cognitive deficiencies may develop over time. Social skills are also affected. All these complications also have a physical response; impairment of physical perception and motor activity, with a loss of automatic movement, is apparent. Patients find it difficult to be aware of the coherent structure of their personality, and they frequently have a poor perception of their own body. The body is an anchor for our personality, and in patients with this disorder, that support disappears (Hátlová, Adámková Ségard, \& Kynštová, 2015).

The benefits of sports were summed up in the systematic review by Soundy, Roskell, Stubbs, Probst, and Vancampfort (2015). Marzolini, Jensen, and Melville (2009) and Beebe et al. (2005) investigated aerobic exercise programmes. The benefits of sports for people with schizophrenia were assessed in systematic review and meta-analysis of Vancampfort et al. (2017). 
Unfortunately, the effectiveness of physical activity and exercise has not yet been adequately compared with other established therapies (Hovland et al., 2013). In their review of exercise therapy for schizophrenia, Gorczynski and Faulkner (2010) underlined the need for larger randomized studies.

The aim of this study was to verify the impact of regularly applied exercise/psychomotor therapy programmes on the mental health of a large sample of participants diagnosed with long-term schizophrenia in in-patient treatment.

\section{Methods}

Before start, this work was approved by the appropriate ethical committee related to the Psychiatric hospital in Prague, Bohnice, in which it was performed. All participants of this study gave informed consent to the work. During the study, all participants followed a routine programme. As a part of the routine programme, there was the monitoring of the mental state of participants. In the case of the negative impact of the study, participation could be immediately stopped. It did not happen in any case.

\section{Participants}

The study included 142 participants (mean age $50.2 \pm 6.51$, age range $21-62 ; 76$ males, mean age $49.2 \pm 6.13$, age range 21-62; 66 females, mean age $51.6 \pm 7.32$, age range 31-61) admitted to the Psychiatric hospital in Prague, Bohnice for in-patient treatment in years 2014-2016. All the participants were diagnosed with schizophrenia according to the DSM 5 and had a history of psychiatric treatment of schizophrenia for more than 3 years, with relatively stable mental health, pharmacotherapy and environment. They were randomly assigned to the experimental group which underwent the intervention $(n=82,44$ males and 38 females) and the control group ( $n=60,32$ males and 28 females). There was no loss to follow-up or discontinued intervention.

\section{Study setting}

The intervention took place in the Psychiatric hospital Bohnice in Prague, patients were exercising in a meeting room of a building for the patients in in-patient care.

\section{Interventions}

The control group underwent a routine programme. The experimental group followed the routine programme and received an exercise programme (psychomotor therapy programme). In addition, all patients needed to follow a scheduled psychotherapy programme.

\section{Routine programme}

The routine programme consisted of pharmacotherapy, memory training (twice a week) and free time (daily).

\section{Psychomotor therapy programme}

The content of the exercise programme was based on previous studies (Hátlová, 2003). The programme had a defined structure that allowed adaptation to the individual level of physical fitness, abilities, and current psychosomatic condition.

The exercise was matched with the actual psychosomatic state, and with a higher level of current abilities of participants. Physical activity was relatively stable; it was altered only to a minimal extent after several foregoing attempts successfully performed. The focus was upon precision in movement structure, as well as on positive emotional experience from the movement mastered. The programme included non-contact exercise and simple gymnastic exercise aiming to the body awareness organized into the Integrative exercise unit, Activation exercise unit, and Relaxation exercise unit. The unit samples are described in more detail by Adámková Ségárd and Hátlová (2012).

The programme was led by two therapists during morning hours and took approximately 45 minutes. It was performed 3 times a week, over a period of 14 weeks.

\section{Outcomes}

To verify the diagnostics and to assess the effects of the psychomotor programme we used The Brief Psychiatric Rating Scale (BPRS; Overall \& Gorham, 1962). The Czech standardized version was published by Filip, Sikora, and Maršálek (1997). The main reason for using BPRS was that it is frequently used rating scale for evaluating the efficacy of treatment, particularly in patients with moderate psychoses. The BPRS contains 18 symptoms, rated on a 7-point scale $(1=$ not present, 7 extremely severe, Czech version uses scores from 0 to 6). Scores are based on a clinician's interview and observation of the patient's behaviour. The scores can be transformed into a factor score: factor 1: anxiety/ depression (ANDP), factor 2: anergy (ANER), factor 3: thought disturbance (THOT), factor 4: activation (ACTV), factor 5: hostility/suspiciousness (HOST).

The BPRS was administered twice, once before the intervention programme and after 14 weeks as a postapplication, every patient was evaluated by three same psychiatric doctors before and after the intervention.

\section{Randomisation}

Participants were randomly assigned to control or experimental group. Each of enrolled participants was matched with a number. This number was used in the 
protocol of randomisation and also for matching tests before and after the intervention.

\section{Blinding}

The data collectors, randomisation providers, data analysts and healthcare providers as psychiatric doctors and nurses were blinded. The head nurse could not be blinded because she had to organize the daily schedule.

Members of the experimental group took a part in an exercise program and there was none offered to the control group.

\section{Statistical methods}

TIBCO Statistica (Version 13.3.0; TIBCO Software, Palo Alto, CA, USA) was used for data processing. To assess for outliers, a comparison of the mean with the median was evaluated by Dixon's Q test. The consistency of the evaluation data between the evaluators was tested by intra-class correlation coefficients. Internal consistency was tested by the Cronbach's coefficient $\alpha$. For statistical comparison, non-parametric statistical methods were used. Normality testing of the data was done by Shapiro-Wilk's test. The analysis was based on the two-step model by Robinson and Levin (1997) requiring beginning with statistical significance assessment followed, in the case of statistically significant results, by calculation of effect size. Because there was non-normal data distribution, to test statistical significance, a Wilcoxon signed-rank test (in case of comparing dependent variables pre-test and post-test) and Mann-Whitney $U$ test (in case of comparing experimental and control group) was used. $P$ values smaller than .01 were considered as significant. The $r$ effect size (Cohen, 1988) was used to calculate the effect sizes for non-parametric data. The $r$ in our study was computed using the formula described in Rosenthal (1994). Cohen's guidelines for $r$ are as follows: small effect $\geq .1$, medium effect $\geq .3$ or large effect $\geq .5$ (Cohen, 1988).

\section{Results}

There were three evaluators (psychiatric doctors). The coefficient consistency of the evaluation data between the evaluators for all the monitored BPRS factors (intraclass correlation coefficient values) exceeded the value of .90. There was good consensus between the evaluators.

In Table 1, descriptive results of the BPRS factors are described for both sexes and groups. The detection of possible outliers using a comparison of means and medians showed no outliers in the data.

The internal consistency of the BPRS was $\alpha=.81$, which is considered as acceptable (Tavakol \& Dennick, 2011).

The comparison of values for the experimental and the control group from the pre-test and the post-test (Table 2) shows that while in the case of the pre-test both groups were comparable (no significant difference)

Table 1

Descriptive characteristics of the factor scores of the Brief Psychiatric Rating Scale, pre- and post-intervention, for the experimental and control group (males and females)

\begin{tabular}{|c|c|c|c|c|c|c|c|c|c|c|c|c|}
\hline & \multicolumn{6}{|c|}{ Male } & \multicolumn{6}{|c|}{ Female } \\
\hline & \multicolumn{3}{|c|}{ Pre } & \multicolumn{3}{|c|}{ Post } & \multicolumn{3}{|c|}{ Pre } & \multicolumn{3}{|c|}{ Post } \\
\hline & $M$ & $M d n$ & $S D$ & $M$ & $M d n$ & $S D$ & $M$ & $M d n$ & $S D$ & $M$ & $M d n$ & $S D$ \\
\hline \multicolumn{13}{|c|}{ Experimental group ( $n=82,44$ male, 38 female $)$} \\
\hline ANDP & 10.46 & 11.17 & 4.04 & 7.09 & 6.33 & 3.99 & 9.48 & 9.50 & 3.50 & 6.26 & 6.33 & 2.90 \\
\hline ANER & 11.89 & 11.00 & 4.71 & 9.48 & 8.33 & 4.71 & 8.95 & 8.33 & 3.24 & 6.67 & 6.83 & 2.99 \\
\hline THOT & 11.21 & 11.50 & 5.09 & 9.22 & 10.00 & 4.85 & 9.33 & 8.67 & 5.69 & 6.95 & 5.50 & 5.67 \\
\hline ACTV & 8.01 & 8.83 & 3.76 & 6.02 & 6.00 & 3.08 & 7.06 & 6.67 & 3.26 & 4.69 & 4.50 & 3.01 \\
\hline HOST & 6.68 & 6.61 & 3.82 & 4.95 & 5.00 & 2.95 & 7.50 & 7.83 & 3.62 & 5.55 & 5.33 & 3.55 \\
\hline \multicolumn{13}{|c|}{ Control group ( $n=60,32$ male, 28 female) } \\
\hline ANDP & 7.98 & 9.67 & 4.83 & 7.67 & 8.83 & 4.89 & 8.80 & 9.00 & 3.98 & 8.56 & 8.83 & 3.94 \\
\hline ANER & 11.86 & 11.00 & 4.36 & 11.52 & 11.00 & 4.33 & 9.30 & 9.67 & 4.04 & 9.31 & 9.33 & 4.01 \\
\hline THOT & 12.57 & 12.00 & 5.25 & 12.11 & 12.00 & 5.53 & 9.44 & 8.83 & 5.54 & 8.77 & 8.67 & 5.64 \\
\hline ACTV & 7.04 & 7.00 & 2.17 & 6.77 & 6.50 & 1.88 & 6.86 & 6.67 & 2.57 & 6.68 & 7.00 & 2.40 \\
\hline HOST & 7.23 & 8.06 & 3.45 & 7.33 & 7.33 & 2.63 & 7.14 & 7.44 & 3.61 & 7.40 & 7.33 & 3.48 \\
\hline
\end{tabular}

Note. $\mathrm{ANDP}=$ Anxiety Depression; ANER $=$ Anergy; THOT $=$ Thought Disturbance $;$ ACTV $=$ Activation; HOST $=$ hostility. 
the post-test values showed statistically significant differences between nearly all variables, except THOT.

A comparison of the data from the BPRS before the intervention programme and 14 weeks after the first evaluation is presented in Table 3.

For the experimental group, the comparison between scores of the BPRS before intervention (pre) and after the intervention (post) revealed significant differences for all factors.

For the experimental group, a large effect size was found for the factors ANDP, ANER, THOT and ACTV between pre- and post-intervention. The factor HOST showed a medium effect size (Table 3 ).

For the control group, the comparison between scores of the BPRS before intervention (pre) and after the intervention (post) did not show any significant differences for any factors, with a significance $p<.01$ (Table 3). A medium effect size was found for THOT in the case of the control group (Table 3).

\section{Discussion}

The current study aimed to explore the impact of psychomotor therapy programmes on the mental health of long-term patients with schizophrenia. While some studies and reviews (Faulkner, 2005; Hölter, 2011;

Table 2

The comparison of values for the experimental and the control group from the pre- and the post-intervention

\begin{tabular}{cccccc}
\hline & \multicolumn{2}{c}{ Pre } & & \multicolumn{2}{c}{ Post } \\
\cline { 2 - 3 } \cline { 5 - 6 } Variable & Male & Female & & Male & Female \\
\hline ANDP & & & & & \\
$p$ & .07 & .77 & & .20 & .01 \\
$r$ & .20 & .00 & & .14 & .32 \\
ANER & & & & .03 & $<.01$ \\
$p$ & .99 & .32 & & .03 & .35 \\
$r$ & .10 & .14 & & .24 & .12 \\
THOT & & & & & .17 \\
$p$ & .33 & .57 & & .04 & \\
$r$ & .10 & .10 & & .24 & .01 \\
ACTV & & & & & .31 \\
$p$ & .10 & .73 & & .33 & $<.01$ \\
$r$ & .32 & .32 & & .49 & .41 \\
HOST & & & & & \\
$p$ & .50 & .84 & .01 & .01 \\
$r$ & .56 & .56 & .70 & .64 \\
\hline
\end{tabular}

Note. $\quad$ ANDP $=$ Anxiety Depression; ANER = Anergy; THOT

$=$ Thought Disturbance; ACTV $=$ Activation, HOST $=$ hostility . $p=$ statistical significance of the Wilcoxon test; $r=$ effect size following the Rosenthal formula.
Probst, Knapen, Poot, \& Vancampfort, 2010; Sampogna et al., 2018; Schuch et al., 2018; Stubbs et al., 2018; Vancampfort et al., 2012) have demonstrated that mental changes may also be achieved through physical activity as a result of removing patients from their immersion in their own world by focusing their attention on physical activity, the causes that may underlie this link are not yet thoroughly studied.

Our results are based, according to the Thornley and Adams (1998), on a large and homogenous sample. They confirmed the existence of a positive impact of psychomotor therapy programmes on mental health. This positive impact was measured by using the BPRS scale, which evaluates five important factors of the behaviour of schizophrenic patients. Using this scale is time-consuming (one evaluation takes about 60 minutes) and requires an experienced psychiatrist. This scale was not used in any previous study evaluating the effect of psychomotor therapy. Design of this study enables the comparison of the standard therapeutic approach and programme enriched by psychomotor therapy. Our results can contribute to the explanation of the findings of extensive research documenting a positive impact of physical activity on self-perception, self-esteem and cognitive functions in psychiatric patients (Beebe et al., 2005; Ellis, Crone, Davey, \& Grogan, 2007; Faulkner, 2005; Gorczynski

Table 3

Inductive analysis of the factor scores of the BPRS preand post-intervention, separately for females and males

\begin{tabular}{|c|c|c|c|c|}
\hline \multirow[b]{2}{*}{ Variable } & \multicolumn{2}{|c|}{ Experimental Group } & \multicolumn{2}{|c|}{ Control group } \\
\hline & Male & Female & Male & Female \\
\hline \multicolumn{5}{|l|}{ ANDP } \\
\hline$p$ & $<.01$ & $<.01$ & .27 & .11 \\
\hline$r$ & .59 & .61 & .23 & .24 \\
\hline \multicolumn{5}{|l|}{ ANER } \\
\hline$p$ & $<.01$ & $<.01$ & .15 & .92 \\
\hline$r$ & .56 & .57 & .20 & .02 \\
\hline \multicolumn{5}{|l|}{ THOT } \\
\hline$p$ & $<.01$ & $<.01$ & .03 & .02 \\
\hline$r$ & .56 & .59 & .30 & .33 \\
\hline \multicolumn{5}{|l|}{ ACTV } \\
\hline$p$ & $<.01$ & $<.01$ & .11 & .11 \\
\hline$r$ & .60 & .61 & .23 & .22 \\
\hline \multicolumn{5}{|l|}{ HOST } \\
\hline$p$ & $<.01$ & $<.01$ & .61 & .32 \\
\hline$r$ & .42 & .48 & .06 & .13 \\
\hline
\end{tabular}

Note. $\quad$ ANDP $=$ Anxiety Depression; ANER = Anergy; THOT $=$ Thought Disturbance; ACTV $=$ Activation, HOST $=$ hostility. $p=$ statistical significance of the Wilcoxon test; $r=$ effect size following the Rosenthal formula. 
\& Faulkner, 2010; Holley, Crone, Tyson, \& Lovell, 2011; Sampogna et al., 2018; Schuch et al., 2018; Simons, Van Damme, Delbroek, \& Probst, 2017; Soundy et al., 2014; Vancampfort et al., 2012; Zamani Sani et al., 2016).

A psychomotor therapy programme applied 3 times a week over 14 weeks showed positive changes in the patients' behaviour in all symptoms of schizophrenia measured by BPRS.

The results achieve a high level of importance, and they support the inclusion of a psychomotor therapy programme in a therapy routine for the treatment of schizophrenia and - as our sample consisted of longterm hospitalized patients - especially for the treatment of patients whose range of problems is difficult to treat using other therapies.

Nonetheless, it is necessary that a programme is tailored and that the participants find these programmes acceptable and interesting. We also recommend working with the closed patient groups which members are nominated by the psychiatrist acquainted with their actual psychosomatic state.

As an explanation of our results, we suggest the improvement in communication and relationship. As in all therapies, the relationship between the patient and the therapist and between patients mutually has important curative potential. This was also the case in our exercise programme, where it was easy to create a positive relationship. This plausible effect could be caused by the fact that participants could move according to their needs and their focus was moved to the exercise. During the exercise programme participants could talk only as much as they like, but they could also exercise quietly. Therefore, verbal communication (thought processes and speech), which is impaired in patients with schizophrenia, was not causing them problems (Hölter, 2011).

It is obvious that when using physically oriented therapeutic methods, it is generally easier to establish contact with mentally ill patients, making this kind of therapy more suitable than most other kinds of therapy (Probst et al., 2010). In other words, when using physical therapy, communication on a non-verbal level may be more understandable and less difficult for the patient (Hátlová, 2003; Hátlová, Adámková Ségard, \& Kynštová, 2015).

Based on previous studies (Rosenbaum et al., 2016; Sampogna et al., 2018; Schuch et al., 2018; Stubbs et al., 2018; Zschucke, Gaudlitz, \& Ströhle, 2013) we could conclude that physical activity and exercise are an effective component of treatment for various mental disorders including schizophrenia.

On the basis of our study, we can confirm the positive influence of psychomotor therapy programme on a large number of participants. As known from the large number of participants we can better count on statistic outcomes. This gives a unique impact of this study on current knowledge about the use of physical activity in schizophrenia treatment.

\section{Limitation of study}

In addition to the satisfying outcomes, one limitation of this study needs to be underlined. The psychomotor therapy programme was run by two therapists whose influence was not assessed.

\section{Conclusions}

The present study verified the relationship between the applied psychomotor therapy programme incorporated into routine therapy and mental health of schizophrenic patients. Our psychomotor therapy programme applied 3 times a week over 14 weeks showed positive changes in the patients' behaviour in all symptoms of schizophrenia measured by BPRS.

On the basis of our outcomes, we believe that we gave strong support to hospital management and therapists to include exercise/psychomotor therapy programmes into a routine programme.

We suggest the exercise/psychomotor therapy programmes as a safe and economically effective treatment for patients with schizophrenia, particularly for those whose range of problems is difficult to treat using standard therapies.

\section{Acknowledgments}

The study was supported by the Ministry of Education, Youth, and Sports of the Czech Republic (Czech-Norwegian Research Program CZ09, grant 7F14500).

\section{Conflict of interest}

There were no conflicts of interest.

\section{References}

Adámková Ségárd, M., \& Hátlová, B. (Eds.). (2012). Psychomotor therapy in schizophrenia treatment. Ústí nad Labem, Czech Republic: Jan Evangelista Purkyně University in Ústí nad Labem.

Beebe, L. H., Tian, L., Morris, N., Goodwin, A., Allen, S. S., \& Kuldau, J. (2005). Effects of exercise on mental and physical health parameters of persons with schizophrenia. Issues in Mental Health Nursing, 26, 661-676. 
Cohen, J. (1988). Statistical power analysis for the behavioral sciences (2nd ed.). Mahwah, NJ: Lawrence Erlbaum Associates.

Ellis, N., Crone, D., Davey, R., \& Grogan, S. (2007). Exercise interventions as an adjunct therapy for psychosis: A critical review. British Journal of Clinical Psychology, 46, 95-111.

Faulkner, G. (2005). Exercise as an adjunct treatment for schizophrenia. In G. Faulkner \& A. Taylor (Eds.), Exercise, health, and mental health: Emerging relationships (pp. 27-47). London, United Kingdom: Routledge.

Filip, V., Sikora, J., \& Maršálek, J. (1997). Praktický manuál psychiatrických posuzovacich stupnic [Practical manual of psychiatric assessment scales]. Prague, Czech Republic: Psychiatrické centrum.

Gaebel, W., \& Falkai, P. (1998). Praxisleitlinien in Psychiatrie und Psychotherapie [Practice guidelines in psychiatry and psychotherapy]. Darmstadt, Germany: Steinkopff.

Gorczynski, P., \& Faulkner, G. (2010). Exercise therapy for schizophrenia. Schizophrenia Bulletin, 36, 665-666.

Harvey, S. B., Hotopf, M., Overland, S., \& Mykletun, A. (2010). Physical activity and common mental disorders. British Journal of Psychiatry, 197, 357-364.

Hátlová, B. (2003). Kinesiotherapy movement therapy in psychiatric treatment. Prague, Czech Republic: Karolinum.

Hátlová, B., Adámková Ségard, M., \& Kynštová, H. (2015). Psychomotor programs in the treatment of schizophrenic. European Health Psychologist, 17, 894.

Holley, J., Crone, D., Tyson, P., \& Lovell, G. (2011). The effects of physical activity on psychological well-being for those with schizophrenia: A systematic review. British Journal of Clinical Psychology, 50, 84-105.

Hölter, G. (2011). Bewegungstherapie bei psychischen Erkrankungen - Grundlagen und Anwendung [Exercise therapy for mental illnesses - basics and application]. Cologne, Germany: Deutscher Ärzte-Verlag.

Hovland, A., Nordhus, I. H., Sjøbø, T., Gjestad, B. A., Birknes, B, Martinsen, E. W., ... Pallesen, S. (2013). Comparing physical exercise in groups to group cognitive behaviour therapy for the treatment of panic disorder in a randomized controlled trial. Behavioral and Cognitive Psychotherapy, 41, 408-432.

Marzolini, S., Jensen, B., \& Melville, P. (2009). Feasibility and effect of a group-based resistance and aerobic exercise program for individuals with severe schizophrenia: A multidisciplinary approach. Mental Health and Physical Activity, 2, 29-36.

Mutrie, N., \& Faulkner, G. (2003). Physical activity and mental health. In T. Everett, M. Donaghy, \& S. Fever (Eds.), Physiotherapy and occupational therapy in mental health. An evidence based approach (pp. 82-97). Oxford, United Kingdom: Butterworth Heinemann.

Overall, J. E., \& Gorham, D. R. (1962). The Brief Psychiatric Rating Scale. Psychological Report, 10, 799-812.

Probst, M., Knapen, J., Poot, G., \& Vancampfort, D. (2010). Psychomotor therapy and Psychiatry: What is in a name? Open Complementary Medicine Journal, 2, 105-113.

Richardson, C. R., Faulkner, G., McDevitt, J., Skirinar, G. S., Hutchinson, D. S., \& Piette, J. D. (2005). Integrating physical activity into mental health services for persons with serious mental illness. Psychiatric Services, 56, 324-331.

Robinson, D. H., \& Levin, J. R. (1997). Research news and Comment: Reflections on statistical and substantive significance, with a slice of replication. Educational Researcher, 26, 21-26.
Rosenbaum, S., Tiedemann, A., Stanton, R., Parker, A., Waterreus, A., Curtis, J., \& Ward, P. B. (2016). Implementing evidence-based physical activity interventions for people with mental illness: An Australian perspective. Australasian Psychiatry, 24, 49-54.

Rosenthal, R. (1994). Parametric measures of effect size. In H. Cooper \& L. V. Hedges (Eds.), The handbook of research synthesis (pp. 231-244). New York, NY: Russell Sage Foundation.

Sampogna, G., Fiorillo, A., Luciano, M., Del Vecchio, V., Steardo, L. Jr., Pocai, B., ... LIFESTYLE Working Group. (2018). A randomized controlled trial on the efficacy of a psychosocial behavioral intervention to improve the lifestyle of patients with severe mental disorders: Study protocol. Frontiers in Psychiatry, 9, 235.

Schuch, F. B., Vancampfort, D., Firth, J., Rosenbaum, S., Ward, P. B., Silva, E. S., ... Stubbs, B. (2018). Physical activity and incident depression: A meta-analysis of prospective cohort studies. American Journal of Psychiatry, 175, 631-648.

Simons, J., Van Damme, T., Delbroek, H., \& Probst, M. (2017). Impact of mental health problems on physical selfesteem. European Psychomotricity Journal, 9, 3-32.

Soundy, A., Freeman, P., Stubbs, B., Probst, M., Coffee, P., \& Vancampfort, D. (2014). The transcending benefits of physical activity for individuals with schizophrenia: A systematic review and meta-ethnography. Psychiatry Research, 220, 11-19.

Soundy, A., Roskell, C., Stubbs, B., Probst, M., \& Vancampfort, D. (2015). Investigating the benefits of sport participation for individuals with schizophrenia: A systematic review. Psychiatria Danubina, 27, 2-13.

Stubbs, B., Vancampfort, D., Smith, L., Rosenbaum, S., Schuch, F., \& Firth, J. (2018). Physical activity and mental health. Lancet Psychiatry, 5, 873.

Tavakol, M., \& Dennick, R. (2011). Making sense of Cronbach's alpha. International Journal of Medical Education, 2011(2), 53-55.

Thornley, B., \& Adams, C. (1998). Content and quality of 2000 controlled trials in schizophrenia over 50 years. British Medical Journal, 317, 1181-1184.

Vancampfort, D., Firth, J., Schuch, F. B., Rosenbaum, S., Mugisha, J., Hallgren, ... Stubbs, B. (2017). Sedentary behaviour and physical activity levels in people with schizophrenia, bipolar disorder and major depressive disorder: A global systematic review and meta-analysis. World Psychiatry, 16, 308-315.

Vancampfort, D., Probst, M., Helvik Skjaerven, L., Catalán Matamoros, D., Lundvik-Gyllensten, A., Gómez-Conesa, A., ... De Hert, M. (2012). Systematic review of the benefits of physical therapy within a multidisciplinary care approach for people with schizophrenia. Physical Therapy, 92, 11-23.

Zamani Sani, S. H., Fathirezaie, Z., Brand, S., Pühse, U., Holsboer-Trachsler, E., Gerber, M., \& Talepasand, S. (2016). Physical activity and self-esteem: Testing direct and indirect relationships associated with psychological and physical mechanisms. Neuropsychiatric Disease and Treatment, 12, 2617-2625.

Zschucke, E., Gaudlitz, K., \& Ströhle, A. (2013). Exercise and physical activity in mental disorders: Clinical and experimental evidence. Journal of Preventive Medicine and Public Health, 46, 12-21. 